\title{
Metal-like ductility and malleability in organic plastic crystals
}

\author{
Amit Mondal, Biswajit Bhattacharya, Susobhan Das, Surojit Bhunia, \\ Rituparno Chowdhury, Somnath Dey and C. Malla Reddy*
}

\author{
Department of Chemical Sciences, Indian Institute of Science Education and Research (IISER) Kolkata, Mohanpur Campus, \\ Mohanpur, Nadia-741246, West Bengal, India.
}

amit.chem.mondal@gmail.com(AM); cmallareddy@gmail.com (CMR)

Ductility, which is a common phenomenon in many metals, is difficult to achieve in molecular crystals. Organic crystals have been recently shown to bend plastically on one or two face specific directions, but they fracture when stressed in any other arbitrary directions. ${ }^{[1]}$ Here, we present an exceptional metal-like ductility and malleability in the isomorphous crystals of two globular molecules, $\mathrm{BH}_{3} \mathrm{NMe}_{3}$ and $\mathrm{BF}_{3} \mathrm{NMe}_{3}$, with characteristic tensile stretching, compression, twisting and thinning (increase of width over $500 \%)^{[2]}$ Surprisingly, the mechanically deformed samples, which transition to lower symmetry phases, not only retain good long range order, but also allow structure determination by single crystal X-ray diffraction. Molecules in these high symmetry crystals interact predominantly via electrostatic forces $\left(\mathrm{B}^{-}-\mathrm{N}^{+}\right)$and form columnar structures, thus forming multiple slip planes with weak dispersive forces among columns. While the former interactions hold molecules together, the latter facilitate exceptional ductility. On the other hand, the limited number of facile slip planes and strong dihydrogen bonding in $\mathrm{BH}_{3} \mathrm{NHMe}_{2}$ negates ductility. The structureproperty correlation established in these aminoboranes with exceptional ductility and ability to retain crystalline order may enable designing highly modular, easy-to-cast crystalline functional organics, for applications in solid-state electrolytes, adaptable electronics $^{[3]}$ (soft ferro/piezo/pyro-electrics), barocalorimetry (solid coolants) ${ }^{[4]}$ soft-robotics etc. ${ }^{[5]}$
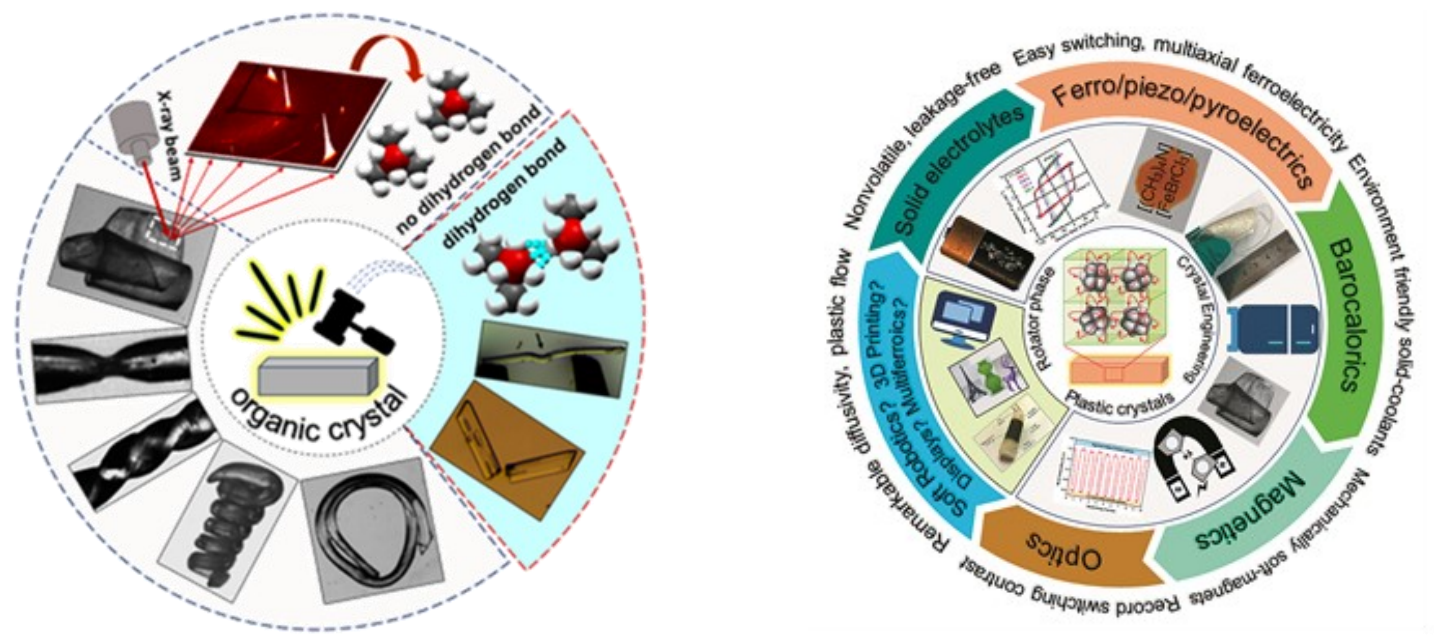

Figure 1. [Left] Peculiar mechanical properties of $\mathrm{BR}_{3} \mathrm{NMe}_{3}(\mathrm{R}=\mathrm{H}, \mathrm{F})$ which show metal like ductility \& malleability along with $\mathrm{X}$ ray diffraction from mechanically yielded thin film and $1 \mathrm{D}$ plasticity in $\mathrm{BH}_{3} \mathrm{NMe}_{3}$ due to presence of dihydrogen bonding. [Right] Emerging applications of plastic crystals in diverse fields like solid-state electrolytes, barocalorimetry, adaptable electronics etc.

[1] Saha, S., Mishra, M. K., Reddy, C. M. \& Desiraju, G. R. (2018). Acc. Chem. Res. 51, 2957-2967.

[2] Mondal, A., Bhattacharya, B., Das, S., Bhunia, S., Chowdhury, R., Dey, S., \& Reddy, C. M. (2020). Angew. Chem. Int. Ed. 59, 2-12.

[3] Harada, J., Shimojo, T., Oyamaguchi, H., Hasegawa, H., Takahashi, Y., Satomi, K., Suzuki, Y., Kawamata, J. \& Inabe, T. (2015). Nat. Chem 8, 946-952.

[4] Li, B., Kawakita, Y., Kawamura, S. O., Sugahara, T., Wang, H., Wang, J., Chen, Y., Kawaguchi, S. I., Kawaguchi, S., Ohara, K., Li, K., Yu, D., Mole, R., Hattori, T., Kikuchi, T., Yano, S. I., Zhang, Z., Zhang, Z., Ren, W., Lin, S., Sakata, O., Nakajima, K. \& Zhang, Z. (2019). Nature, 567, 506-510

[5] Das, S., Mondal, A. \& Reddy, C. M. (2021). Chem. Soc. Rev. 49. 8878-8896.

Keywords: crystal engineering; organic plastic crystals; ductility \& malleability; adaptable electronics; dihydrogen bonding Acta Cryst. (2021), A77, C1224 